\title{
Efficient Shock Wave Coupling to a Waveguide
}

\author{
Ihalainen, Peetu
}

IEEE

2019

Ihalainen , P , Malinen , H, Heikkilä , J , Veira Canle , D , Kontiola , A, Salmi , A \& Haeggström , E 2019 , Efficient Shock Wave Coupling to a Waveguide . in 2019 IEEE International Ultrasonics Symposium (IUS) . IEEE International Ultrasonics Symposium, IEEE , pp. 170-172, IEEE International Ultrasonics Symposium , Glasgow , United Kingdom , 06/10/2019 . https://doi.org/10.1109/ULTSYM.2019.8925561

http://hdl.handle.net/10138/326117

https://doi.org/10.1109/ULTSYM.2019.8925561

acceptedVersion

Downloaded from Helda, University of Helsinki institutional repository.

This is an electronic reprint of the original article.

This reprint may differ from the original in pagination and typographic detail.

Please cite the original version. 


\section{Efficient Shock Wave Coupling to a Waveguide}

\author{
Peetu Ihalainen \\ Electronics Research Lab, \\ Department of Physics \\ University of Helsinki \\ Helsinki, Finland \\ peetu.ihalainen@helsinki.fi
}

\author{
Antti Kontiola \\ University of Helsinki \\ Helsinki, Finland
}

\author{
Henri Malinen \\ Electronics Research Lab, \\ Department of Physics \\ University of Helsinki \\ Helsinki, Finland
}

\author{
Jesse Heikkilä \\ Electronics Research Lab, \\ Department of Physics \\ University of Helsinki \\ Helsinki, Finland
}

\author{
Daniel Veira Canle \\ Electronics Research Lab, \\ Department of Physics \\ University of Helsinki \\ Helsinki, Finland
}

\author{
Ari Salmi \\ Electronics Research Lab, \\ Department of Physics \\ University of Helsinki \\ Helsinki, Finland
}

\author{
Edward Hæggström \\ Electronics Research Lab, \\ Department of Physics \\ University of Helsinki \\ Helsinki, Finland
}

\begin{abstract}
High-voltage induced shock waves were created in different shaped spark chambers. The generated shock waves were imaged after they had traveled through a waveguide. Imaging was done by using a stroboscopic schlieren method to determine the speed of the shock wave in air. The effect of the spark chamber shape as well as the effect of the width of the spark gap were studied. The simple cylindrical chamber had the highest efficiency of the measured geometries. A wider spark gap resulted in a higher overpressure of the shock wave.
\end{abstract}

Keywords-Non-linear acoustics, Shock waves, Schlieren

\section{INTRODUCTION}

Non-linear acoustic waves are useful for actuating on a sample in a non-contacting manner[1]. Compared to linear acoustic waves, shock waves propagate at increased speed and deposit their energy quickly into the sample. To direct shock waves one can employ a waveguide so that they impinge normally on the surface of the sample thus maximizing the momentum transfer.

Guided linear waves that focus a shock wave have been used in medicine to treat patients with uroliths in the bladder[2].

This study shows how tailoring the shape of the spark chamber impacts the pressure of the delivered shock wave at the end of the waveguide. There is research work on how to focus shock waves inside an elliptic structure [3], but no earlier research exist on focusing shock waves into a waveguide.

\section{METHODS}

\section{A. Electronics}

We produced shock waves by discharging a $1 \mu \mathrm{F}$ capacitor charged to $1.8 \mathrm{kV}(1.62 \mathrm{~J})$ between two tungsten electrodes. The capacitor discharge was precisely timed by ionizing the space between the electrodes with a smaller discharge. This small ionizing spark was created by discharging a small capacitor to the ground with a thyristor. A computer synchronized the firing of the discharge with the imaging system.

The capacitor discharge creates a plasma spark that generates a shock wave. This perturbation is guided by the structure of the spark chamber into the waveguide (Fig. 1).

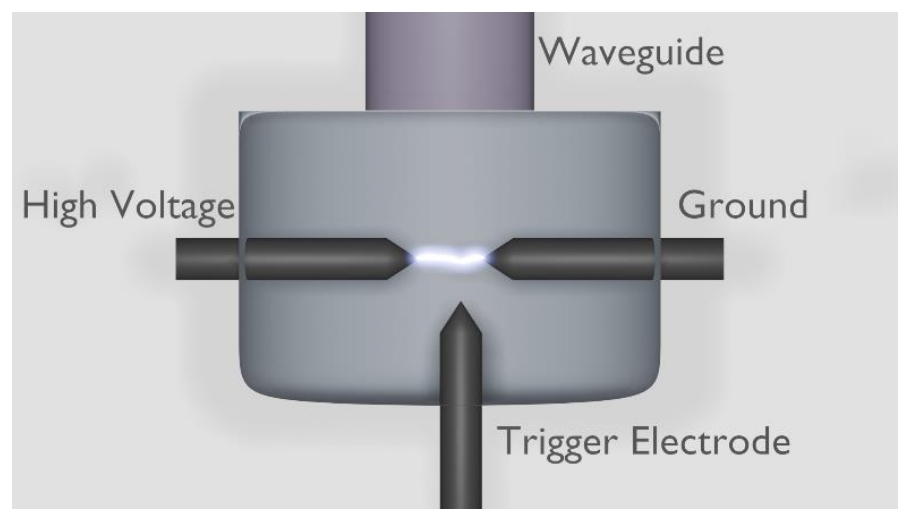

Fig. 1. Schematic representation of the electrode placement inside the spark chamber.

\section{B. Waveguide and the spark chamberaa}

The waveguide was a flexible tube made of polyurethane. The inner diameter of the waveguide was $6.5 \mathrm{~mm}$ and its crosssection was circular. We imaged the shock waves with different time delays (i.e. at different locations).

Different chamber geometries were tested to understand the impact of the spark chamber shape on the shock wave coupling into the waveguide. The waveguide was kept the same. Consequently attenuation that was caused by the tube was same for all of the different chambers. 
The effect of the placement of the high voltage electrode relative to the ground electrode was investigated. This was done by altering the distance between the high-voltage electrode and the ground electrode.

During the measurements three tungsten electrodes were attached to each of chamber. The same electrodes were used in all chambers to avoid differences caused by the shape or the structure of the electrodes. Each chamber was tested using some specific electrode distance which allowed comparison between the chamber for each spark gap width.

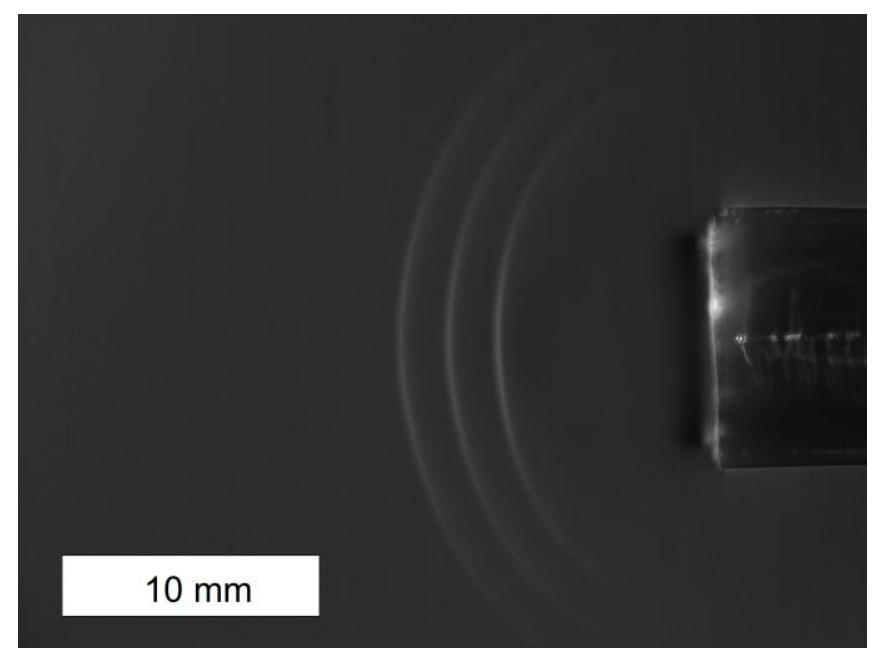

Fig. 2. Propagating shock wave $(383 \mathrm{~m} / \mathrm{s})$ imaged using stroboscopic schlieren method. The waveguide is seen in the image at the right edge.

\section{Measuring the efficiency}

The efficiency of each spark chamber was determined by comparing the overpressure of the generated shock waves. Nonlinear waves propagate faster at higher pressures. By calculating the propagation speed, we determined the pressure generated by different chamber designs.

The following equation links the shock wave speed to its pressure[5]:

$$
p_{o}=\frac{2 \gamma M^{2}-(\gamma-1)}{\gamma+1} p-p
$$

Here $p$ is the static pressure of the fluid, $p_{o}$ is the overpressure and $\gamma$ is the ratio of specific heats, in case of air $\gamma=1.4$. $M$ is the dimensionless Mach number of the flow which describes the speed of the shock wave compared to the constant speed of sound in the medium; $\mathrm{M}=\mathrm{v} / \mathrm{c}$.

The measurements were done using a stroboscopic schlieren imaging method. The field of view was illuminated with three cycles of $200 \mathrm{kHz}$ square wave driving a high-power LED. This yields images that feature three shock wave fronts separated by $5 \mu \mathrm{s}$ in the time domain (Fig. 2 and 3). Timing the spark generation and the image acquisition allows us to determine the speed of the shock wave at different locations after the shock has exited the waveguide.

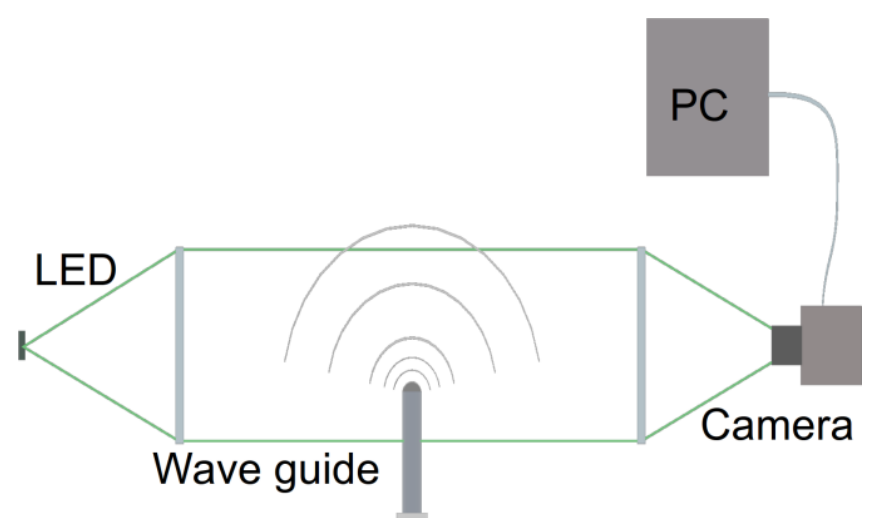

Fig. 3. Schematic representation of the schlieren set-up

\section{Chamber Geometries}

Four different geometries for the chamber were tested during the measurements. The shapes included one cylindrical chamber (C), two different parabolic focusing shapes (A, B) and one chamber that had rocket nozzle like shape (D). With chambers $\mathrm{A}$ and $\mathrm{B}$ the breakdown takes place close to the focal point of the parabola and the waveguide is attached so that the other focal point is just inside the waveguide. These geometries are shown in Fig. 3 and are later referred to with their letters.

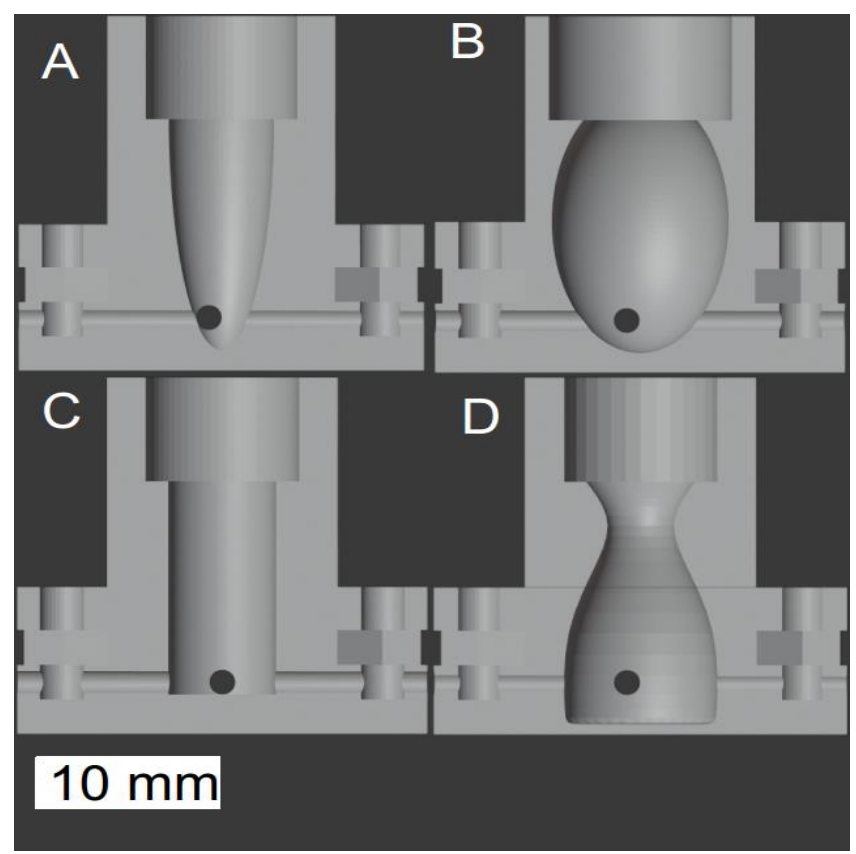

Fig. 4. Cross-sections of the spark chambers that were used in the measurements. The channels for the electrodes can be seen in the bottom part.

\section{RESULTS}

\section{A. Differences between the geometries}

Chambers $\mathrm{A}, \mathrm{B}$ and $\mathrm{C}$ show no major difference in shock wave generation efficiency, however chamber $D$ had significantly worse efficiency (Fig. 4). The figure shows the case when the spark gap is $4 \mathrm{~mm}$. There was no noticeable increase in maximum overpressure of the shock wave created in the focusing structures compared to the non-focusing counterpart, sample $\mathrm{C}$. 


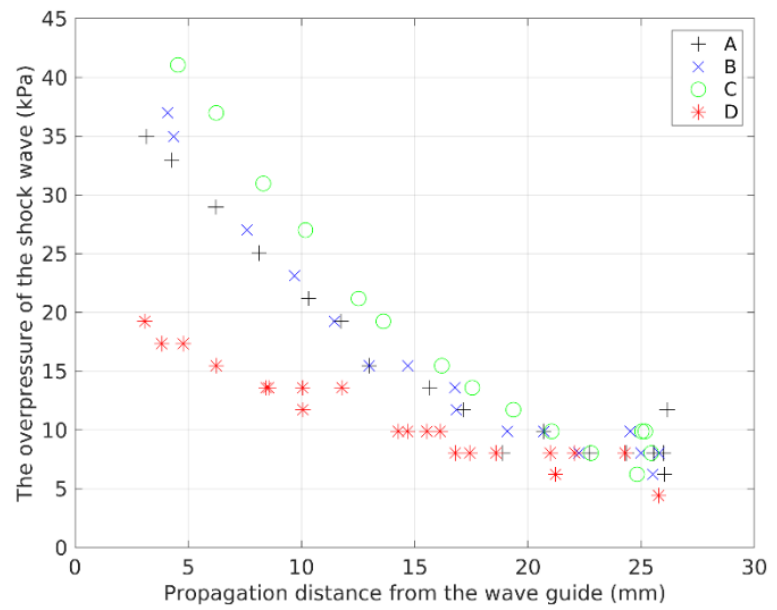

Fig. 5. Overpressure of the shock waves generated by different chamber geometries as a function of distance from the waveguide.

\section{B. Effect of the distance between the electrodes}

The bigger the distance between the electrodes the higher the overpressure of the shock wave (Fig. 5). Although a wider spark gap increased the overpressure of the shock wave, the probability of the breakdown taking place and thus generating the shock wave was reduced. The spark gap can be optimized for the highest possible overpressure without sacrificing triggering reliability. The optimal spark gap distance depends on the voltage used in the measurements.

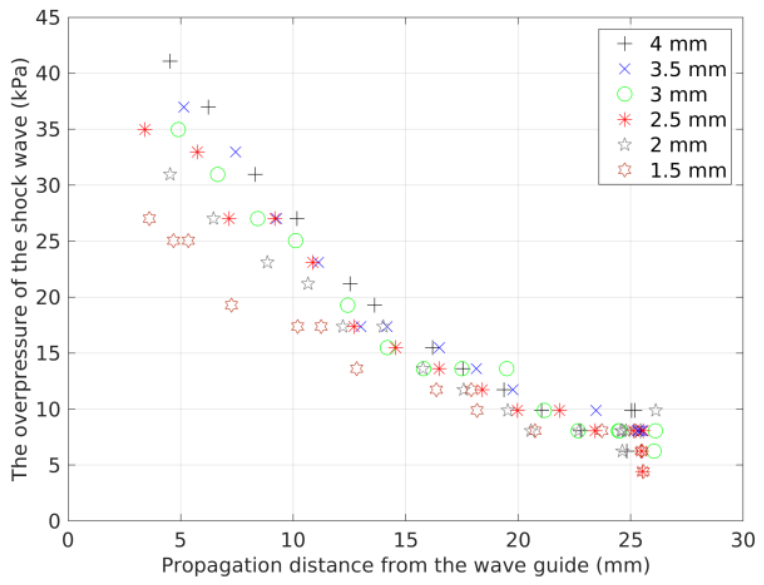

Fig. 6. Effect of the spark gap distance on the overpressure of the shock wave (Cylindrical Chamber, C)

\section{Secondary features}

Two of the chambers (B, D) created secondary shock waves which were captured in the schlieren images (Fig 6). Especially chamber D created a remarkable secondary shock wave trailing the primary shock wave. The analysis that evaluated the overpressure only focused on the primary shock wave. There is an air vortex visible in each image just after the waveguide.

When these kinds of structures are used to actuate samples, one should take into account possible other features in addition to the primary shock wave.

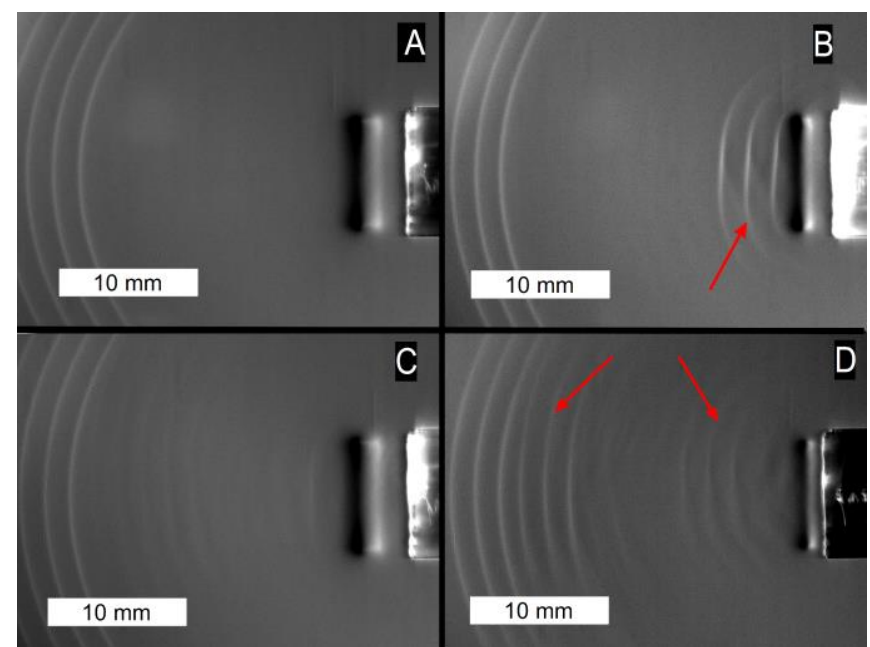

Fig. 7. Additional features seen with different chamber geometries. Extra shock waves are indicated with red arrows.

\section{DISCUSSION}

The efficiency of the spark chamber geometries was studied experimentally. Focusing the shock wave into a waveguide by means of parabolic reflectors did not increase the overpressure and the highest overpressure was reached by using a cylindrical chamber.

The electrode placement had a significant effect on the overpressure of the generated shock wave. The spark gap width had an upper limit after which the shock wave generation became impossible.

\section{CONCLUSION}

Advanced shock wave chamber shapes seem to provide little benefit to the maximum overpressure obtained, and thus, simple geometries are preferred. The electrode placement has an impact on the overpressure of the shock wave when producing shock waves using this method.

\section{ACKNOWLEDGMENT}

Thank you, B.Sc. Eetu Lampsijärvi for building the schlieren set-up and B.Sc. Joni Mäkinen for proofreading this paper.

\section{REFERENCES}

[1] J. Heikkilä, E. Lampsijärvi, A. Kontiola, A. Salmi, E. Hæggström, Nonlinear-wave-based excitation of waves on a membrane, 2019, unpublished

[2] Schedin, S., Gren, P. \& Wåhlin, A. Shock Waves (1997) 7: 343

[3] Adams \& Senior, Electrohydraulic and extracorporeal shock-wave lithotripsy, Veterinary Clinics: Small Animal Practice, Volume 29, Issue $1,293-302$

[4] AMES Research Staff, Report 1135 Equations, Tables, and Charts For Compressible Flow, AMES Aeronautical Laboratory,1953, eq. 93 